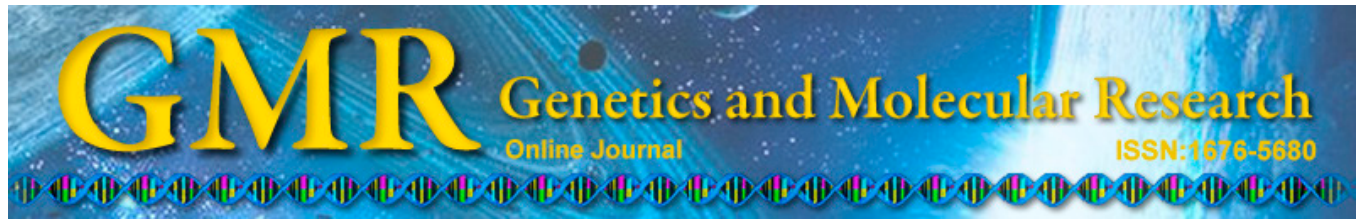

\title{
Wnt1-induced MAFK expression promotes osteosarcoma cell proliferation
}

\author{
R. Wang, J. Zheng, D.-S. Zhang, Y.-H. Yang and Z.-F. Zhao \\ Department of Orthopedics, The 117th Hospital of PLA, Hangzhou, Zhejiang, \\ China
}

Corresponding author: Z.-F. Zhao

E-mail: zhaozfag@126.com

Genet. Mol. Res. 14 (3): 7315-7325 (2015)

Received July 3, 2014

Accepted November 13, 2014

Published July 3, 2015

DOI http://dx.doi.org/10.4238/2015.July.3.7

ABSTRACT. Osteosarcoma is one of the most common primary bone tumors in children and young adults. In this study, we investigated the role of musculoaponeurotic fibrosarcoma oncogene homolog $\mathrm{K}$ (MAFK) in osteosarcoma cell proliferation in vitro and the possible pathways that contributed to MAFK-related osteosarcoma development. We first reported that $M A F K$ was expressed at low levels in an osteosarcoma cell line. Furthermore, a significant correlation between MAFK and the Wnt signaling pathway was observed in osteosarcoma by using a gene microarray assay. We found that expression of MAFK could be induced by Wnt1 in a dose-dependent manner. Furthermore, Wnt1-induced MAFK expression caused a significant increase of cell viability, whereas a Wnt pathway inhibitor, IWR-1-endo, abolished Wnt1-induced effects on MAFK. Finally, cell cycle analysis showed that enhanced cell proliferation might be attributed to re-distribution of the cell cycle. Together, our results suggested that Wnt1-induced MAFK expression promoted cell proliferation in MG63 cells, and that the role of MAFK in osteosarcoma might be closely linked to the Wnt signaling pathway.

Key words: Wnt; MAFK; Osteosarcoma; Proliferation 


\section{INTRODUCTION}

Osteosarcoma is recognized as one of the most common primary bone tumors in children and young adults, exhibiting preferred localization in the metaphysic of the long bones in adolescents (Broadhead et al., 2011). To date, standard therapies for osteosarcoma include surgical resection as well as neoadjuvant chemotherapy (Chou et al., 2008). It has been reported that surgery alone could cure $11 \%$ of patients suffering from osteosarcoma, whereas the combination of surgery with chemotherapy significantly improved the survival rate up to $60-70 \%$ (Chou et al., 2008; Trent, 2008). Unfortunately, chemotherapeutics inevitably cause adverse effects to patients and are considered toxic to varying degrees (Siegel and Pressey, 2008). In addition, despite recent improvements in osteosarcoma treatments, morbidity and mortality rates of highly aggressive osteosarcoma remain high. Approximately $32-49 \%$ of patients who are diagnosed with high-grade osteosarcoma can statistically expect a life expectancy of less than 60 months (Bielack et al., 2008). Thus, identification of novel therapies specific for osteosarcoma is a crucial step forward for the treatment and prevention of highly aggressive tumors (Chou and Gorlick, 2006; Siegel and Pressey, 2008).

Recent discoveries of targeted molecular therapies have greatly advanced our understanding of human cancer treatments (Ghosh et al., 2014; Gorjanacz, 2014). In addition, a better comprehension of oncogene families such as musculoaponeurotic fibrosarcoma (MAF) has aroused the interests of scientists for additional research into their roles in human disease.

The MAF oncogene family has expanded from the original discovery of the founding member v-Maf, which was identified during analysis of the avian transforming retrovirus initially isolated from chicken (Nishizawa et al., 1989). MAFs are members of the basic leucine zipper (bZIP) family that possess both evolutionarily conserved basic and leucine zipper domains. In general, the basic domain primarily binds to DNA, whereas the leucine zipper domain mediates homo- or hetero-dimerization with specific bZIP transcription factors, including AP-1, CREB/ATF, CNC, C/EBP, and PAR proteins (Vinson et al., 2006). This plethora of binding partners suggests the existence of complex networks of MAFs in gene regulation.

v-maf avian musculoaponeurotic fibrosarcoma oncogene homolog K (MAFK), a small protein of the MAF family, is localized predominantly in the nucleus with a molecular weight about approximately 18 kDa (Blank et al., 1997; Francastel et al., 2001; Brand et al., 2004). MAFK is of considerable clinical importance, as it has been widely reported to be involved in multiple diseases, including diabetes, neuronal disorders, thrombocytopenia, and carcinogenesis (Kannan et al., 2012). In particular, MAFK was thought to be able to maintain redox homeostasis due to its function in antioxidant response; overexpression of MAFK led to a dose-dependent repression of antioxidant response element (ARE) activity (Nguyen et al., 2000). In fact, the role of MAFK was functionally linked to NF-E2-related factor 2 (Nrf2). Interactions of MAFK with Nrf2 have been previously described (Ney et al., 1993; Nguyen et al., 2000), and additional studies have suggested that the Nrf2/MAFK complex was involved in transcriptional activation/repression mediated by the ARE (Itoh et al., 1997). However, whereas Nrf2 was shown to activate ARE transcriptional activity in HepG2 cells, MAFK repressed ARE activity (Nguyen et al., 2000). MAFK-Nrf2 heterodimers were shown to play a role in negative regulation of ARE-mediated transcription and in antioxidant induction of NQO1 (a downstream gene of Nrf2) and other detoxifying enzyme genes (Dhakshinamoorthy and Jaiswal, 2000). However, recent experiments have also utilized the growth factor- $\beta$ induced transcription factors MAFK and BACH1 to suppress expression of the heme oxygen- 
ase-1 gene (Okita et al., 2013), indicating that MAFK is also under the strict control of other molecules in regulating disease progression. Interplay between MAFK and other molecules also suggests that MAFK might be widely involved in human disease through a series of regulatory mechanisms.

A recent study reported that MAFs promoted osteoblast differentiation in mice by mediating the age-related switch in mesenchymal cell differentiation (Nishikawa et al., 2010). One interesting question that arose from this study was whether MAFK could play the role in osteosarcoma as observed in osteoblast differentiation. Thus, our study aimed to investigate the expression profile of MAFK and its role in osteosarcoma. In addition, we sought to examine the possible pathways involved in this process using microarrays.

\section{MATERIAL AND METHODS}

\section{Reagents}

Wnt1 and MAFK cDNA fragment was generated by PCR. The amplified fragments were digested with HindIII and XhoI (Takara, Dalian, China) and inserted into HindIII and XhoI sites of the pcDNA4 vector (Invitrogen). The expression plasmids were verified by DNA electrophoresis and sequencing. The Wnt signaling pathway inhibitor, IWR-1-endo (Chen et al., 2009), was obtained from Cayman Chemical Co. (Ann Arbor, MI, USA). Dimethylsulfoxide (DMSO) was obtained from Sigma-Aldrich (St. Louis, MO, USA), Dulbecco's modified Eagle medium (DMEM) was obtained from Corning (Manassas, VA, USA), and fetal bovine serum (FBS) was obtained from Thermo Fisher Scientific (Waltham, MA, USA). Primary antibodies against MAFK and glyceraldehyde 3-phosphate dehydrogenase (GAPDH) were purchased from Santa Cruz Biotechnology (Santa Cruz, CA, USA).

\section{Cell line and cell culture}

The human osteosarcoma cell line MG63 was purchased from the American Type Culture Collection (Manassas, VA, USA). Cells were cultured in DMEM supplemented with $10 \% \mathrm{FBS}$ at $37^{\circ} \mathrm{C}$ in a $5 \% \mathrm{CO}_{2}$ humidity-controlled incubator.

\section{Transient transfection}

Transient transfection of plasmids was conducted using Lipofectamine 2000 reagent (Invitrogen Corporation, Carlsbad, CA, USA) according to manufacturer protocol. Briefly, 24 h post-transfection, MG63 cells were washed with phosphate-buffered saline (PBS) and refed with fresh medium. Forty eight hours after transfection, the cells were harvested and transfection efficiency was verified by western blotting.

\section{Quantitative real-time PCR (qRT-PCR)}

Total RNA was extracted from MG63 cells using Trizol Reagent (Sigma-Aldrich). For quantification of mRNA levels, total RNA $(1 \mu \mathrm{g})$ was reverse-transcribed using $200 \mathrm{U}$ SuperScript II Reverse Transcriptase (Invitrogen) and 500 ng Oligo (dT) primers to generate cDNAs. cDNAs were then subjected to qPCR assays using iTaq Fast SYBR Green Su- 
permix with ROX (Bio-Rad Laboratories; Hercules, CA, USA), $0.2 \mu \mathrm{M}$ primers (MAFK F: 5'-GCACACATGGCAGAGAGAGT-3', R:5'-GAGTCCTGCTCACCGTCAAA-3'; GAPDH F:5'-CTCTGCTCCTCCTGTTCGAC-3', R:5'- CACCTTCCCCATGGTGTCTC-3') with the 7500 Fast Real-Time PCR System (Applied Biosystems, Inc., Foster City, CA, USA). PCR cycling conditions were: $95^{\circ} \mathrm{C}$ for $29 \mathrm{~s}$, followed by 38 cycles of $95^{\circ} \mathrm{C}$ for $5 \mathrm{~s}$ and $60^{\circ} \mathrm{C}$ for 34 s. Amplification specificity was evaluated by melting curve analysis. Relative mRNA levels were determined using the formula $2^{-\Delta \mathrm{CT}}(\mathrm{CT}$; cycle threshold) where $\Delta \mathrm{CT}=\mathrm{CT}$ (target gene) - CT (GAPDH). All assays were performed in triplicate.

\section{Western blot analysis}

Forty eight hours after transfection, the cells were harvested and solubilized with lysis buffer with complete protein inhibitor cocktail (Roche). Protein concentrations were determined using the BCA Protein kit (Thermo Fisher Scientific). Thirty milligrams protein was size-fractionated using 10\% gradient sodium dodecyl sulfate-polyacrylamide gel electrophoresis Ready-Gels (Bio-Rad Laboratories) before electroblotting onto nitrocellulose membranes. Membranes were then blocked with 5\% skim milk in 0.1\% Tween 20 (Sigma-Aldrich) Tris-buffered saline (TBST). MAFK and GAPDH primary antibodies were diluted (1:1000) in TBST with $5 \%$ milk prior to use. Primary antibody incubations were then performed overnight at $4{ }^{\circ} \mathrm{C}$ after antigen blocking. Thereafter, membranes were washed with TBST and incubated in the corresponding horseradish peroxidase-conjugated secondary antibodies at $37^{\circ} \mathrm{C}$ for 1 h. The blotted membranes were treated using the SuperSignal West Dura Extended Duration Substrate (Pierce Biotechnology Inc.), and signals were detected using a Las-4000 mini CCD camera (GE Healthcare). GAPDH was used as a loading control.

\section{Immunofluorescence analysis}

Cells pretreated with 1, 10, and $100 \mu \mathrm{M}$ Wnt1 (Sigma-Aldrich, St. Louis, MO, USA) were cultured in Lab-Tec chamber slides (Nalge Nunc, Roskide, Denmark), fixed with 4\% paraformaldehyde/PBS, and permeabilized with $0.1 \%$ Triton X-100/PBS. Slides were blocked with $3 \%$ goat serum in $1 \%$ bovine serum albumin/PBS at $37^{\circ} \mathrm{C}$ for $30 \mathrm{~min}$, incubated with anti-MAFK antibody $(1: 100)$ overnight at $4^{\circ} \mathrm{C}$, then incubated with Alexa Fluor 594 donkey anti-mouse $\operatorname{IgG}(1: 200)$ at $37^{\circ} \mathrm{C}$ for $1 \mathrm{~h}$. Signals were visualized using a laser-scanning microscope (Axiovert 100M, LSM 510 system; Carl Zeiss Japan, Tokyo, Japan). MG63 cells pretreated with DMSO were used as the control group.

\section{Gene set enrichment analysis (GSEA)}

To investigate the possible pathway that contributed to MAFK-mediated osteosarcoma carcinogenesis, we use GSEA to analyze the microarray data from genechip database (Gene Expression omnibus). The Wnt pathway in pancreatic cancer was used as a negative control. Data from the scanned arrays were extracted by the Genome Studios software (v. 1.6, Illumina, San Diego, CA, USA). Data processing and statistics were performed using Matlab (The Mathworks, Natick, MA, USA), as was multidimensional scaling analysis of the detected probes. For gene analysis, microarray data were analyzed with the filtering criteria of adjusted $\mathrm{P}<0.05$ and $>15 \%$ change. To reveal the genes with significantly changed expression, 
heatmap analysis was used for visualization in Matlab. Pathway analysis was performed using Ingenuity Pathway Analysis (Ingenuity Systems, Redwood City, CA, USA) and the false discovery rate Benjamini Hochberg algorithm $\mathrm{P}<0.05$ was subsequently used to determine statistical significance.

\section{Cell counting kit-8 (CCK-8) cell proliferation assay}

Two days after treatment (DMSO, MAFK, MAFK+IWR-1-edo, MAFK+Wnt-1), MG63 cells were trypsinized and $3 \times 10^{3}$ cells were plated on a 96-well plate per well. This time point was set as day 1 . Cell viability was determined each day by adding $10 \mu \mathrm{L}$ CCK-8 (tetrazolium salt) reagent (Dojindo, Kumamoto, Japan) to each well. After incubation at $37^{\circ} \mathrm{C}$ for $4 \mathrm{~h}$, the absorbance at $450 \mathrm{~nm}$ was measured. Absorbance represented cell numbers of each group of cells. A continuous observation over 4 days was conducted.

\section{Fluorescence-activated cell sorting (FACS) assay}

After distinct treatments (DMSO, MAFK, MAFK+IWR-1-edo, MAFK+Wnt-1) to MG63 cells for $48 \mathrm{~h}$, cells were harvested and fixed with $70 \%$ ethanol at $-20^{\circ} \mathrm{C}$, followed by incubation with $50 \mu \mathrm{g} / \mathrm{mL}$ propidium iodide (Molecular Probes, Eugene, OR, USA) and $1 \mu \mathrm{g} /$ $\mathrm{mL}$ RNase A for $30 \mathrm{~min}$ at room temperature. DNA contents were then analyzed using a FACS Caliber system (Becton Dickinson, Bedford, MA, USA). The distribution of cells in each cellcycle phase was determined with the ModFitLT Software (Becton Dickinson).

\section{Statistical analysis}

Data shown represent at least three independent replicate experiments performed in triplicate and are reported as means \pm standard deviation. Statistical significance was determined by the Student $t$-test with significance set at $\mathrm{P} \leq 0.05$.

\section{RESULTS}

\section{MAFK was expressed at low levels in osteosarcoma}

To initially determine the expression profile of MAFK in osteosarcoma, we detected $M A F K$ mRNA in paired non-cancerous bone tissue and osteosarcoma. mRNA levels were normalized to GAPDH expression, which was selected as an internal reference gene. As shown in Figure 1, normal bone exhibited an average relative value of 9, whereas MAFK expression in the osteosarcoma cell line MG63 only exhibited an average value of approximately 7.5. The mRNA level of $M A F K$ was significantly decreased $(\mathrm{P}=0.024)$ in osteosarcoma as compared with normal tissue.

\section{Role of MAFK in osteosarcoma was found to be closely linked to the Wnt signaling pathway}

To decipher the possible pathways that contributed to MAFK modulation, we used GSEA analysis. Interestingly, we found that MAFK was significantly linked to the Wnt signal- 
ing pathway in osteosarcoma $(\mathrm{P}=0.004)$ (Figure 2A). As the Wnt signaling pathway is abnormally active in pancreatic cancer, we utilized this pathway in pancreatic cancer as a control. As expected, no significant correlation of MAFK with Wnt signaling was observed in the control group $(\mathrm{P}=0.07)$ (Figure $2 \mathrm{~B}$ ). Figure $2 \mathrm{C}$ and $\mathrm{D}$ also shows that MAFK was significantly correlated with the Wnt signaling pathway in osteosarcoma, as evidenced by a broad spectrum of genes from the Wnt pathway with altered expression levels. As an approach to identify genes and pathways that are involved in disease development, heatmap analysis was conducted. The heatmap analysis showed that multiple genes involved in Wnt signaling were elevated, whereas many other genes were also decreased in osteosarcoma (Figure 2E). In all, these data suggested that MAFK is closely related to the Wnt signaling pathway in osteosarcoma.

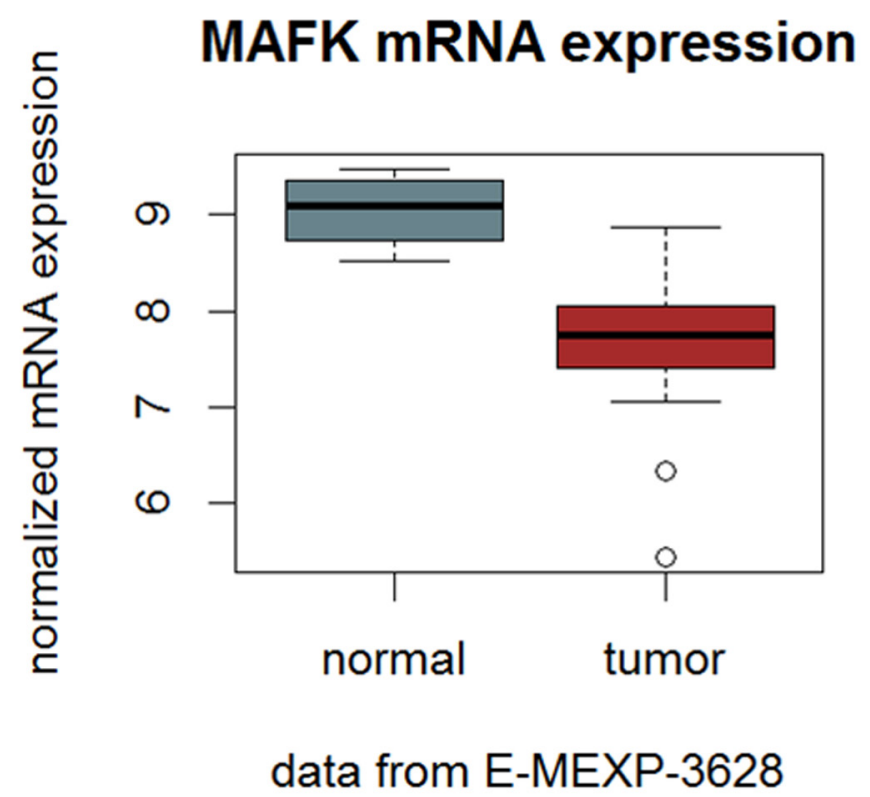

Figure 1. Low levels of $M A F K$ expression in osteosarcoma. The mRNA level of $M A F K$ was determined by quantitative real time polymerase chain reaction amplification (qRT-PCR), using GAPDH as an internal reference gene.

\section{Wnt1 induced MAFK expression in MG63 cells}

Based on the above observations, we speculated that MAFK is involved in osteosarcoma in the presence of Wnt signaling molecules. In this study, we selected Wnt1 as a representative gene from the Wnt signaling pathway to further uncover the detailed network between MAFK and the Wnt pathway. To examine this question, we initially conducted immunofluorescence analysis. Because MAFK was observed to be expressed at low levels in osteosarcoma, we constructed a MAFK expression plasmid to artificially increase the MAFK level in MG63 cells. MG63 cells were pretreated with 1, 10, and $100 \mu \mathrm{M}$ Wnt1, and cells treated with DMSO were cultured as controls. Compared to control MG63 cells, we found that $1 \mu \mathrm{M}$ Wnt1-treated MG63 cells exhibited an increase in MAFK expression. In addition, along with increased Wnt1 concentration, expression of MAFK was accordingly increased (Figure $3 \mathrm{~A})$. We then determined the mRNA level of $M A F K$ after Wnt 1 treatments. Consistently, we 
found that the mRNA level of $M A F K$ was increased by two-fold in the $1 \mu \mathrm{M}$ Wnt1-treated group. When Wnt1 was at $100 \mu \mathrm{M}$, the mRNA level of MAFK exhibited an approximately 3.5-fold increase (Figure 3B). Comparable results were also observed after quantification of western blotting (Figure 3C). Taken together, the results suggested that the expression level of MAFK could be induced by Wnt1 in a dose-dependent manner.

A

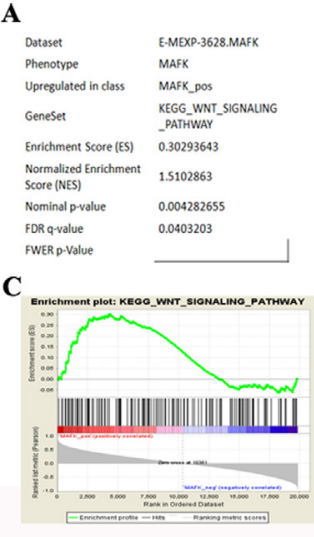

B

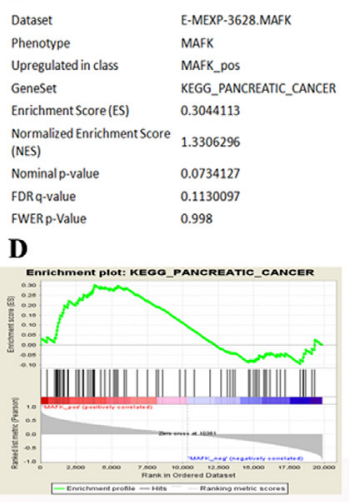

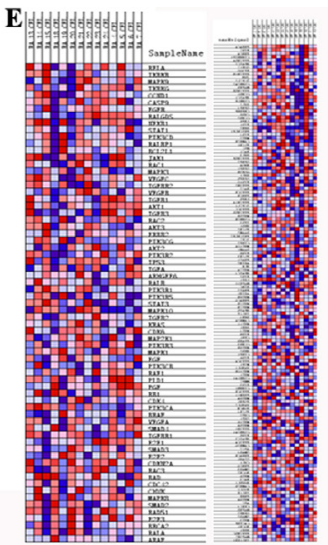

Figure 2. Close linkage of MAFK to the Wnt signaling pathway in osteosarcoma. A. B. MAFK was shown to be significantly related to the Wnt signaling pathway in osteosarcoma using KEGG database analysis $(\mathrm{P}=0.004)$. Results for the Wnt pathway in pancreatic cancer were used as a control. C. D. Multiple genes from the Wnt pathway were shown to be modulated in MAFK-related osteosarcoma samples. E. Heatmap analysis showed that a broad spectrum of genes from the Wnt signaling pathway exhibited increased expression in osteosarcoma as compared with normal tissue, whereas the expression of others was decreased.

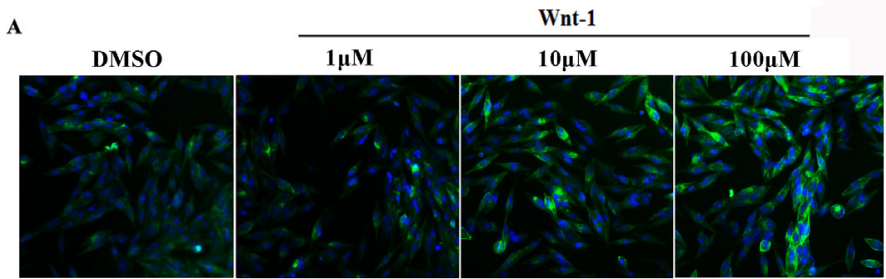

B

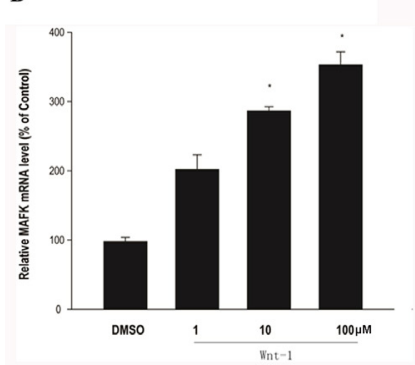

C

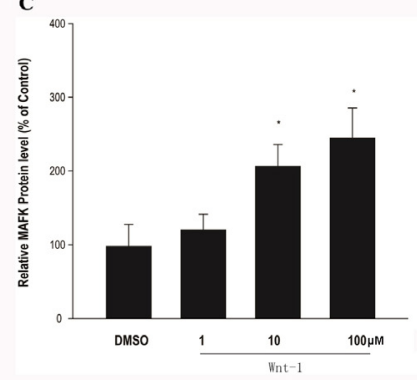

Figure 3. Induction of $M A F K$ expression in MG63 cells by Wnt1. A. Immunofluorescence analysis revealed altered $M A F K$ expression in response to Wnt 1cultured. B. Quantitation of MAFK mRNA demonstrated that $M A F K$ expression could be elevated by Wnt1 in a dose-dependent manner. C. Comparable results were also observed by quantification of MAFK protein levels using western blotting. ${ }^{*} \mathrm{P}<0.05$. 


\section{Wnt1-induced MAFK expression promoted cell proliferation in MG63 cells}

To functionally investigate the effects of Wnt1-induced MAFK expression on osteosarcoma development, MG63 cells were treated with MAFK alone, or together with Wnt1 expression plasmids (MAFK+Wnt1 group). The Wnt signaling pathway inhibitor, IWR-1-endo, was also used (MAFK+IWR-1-endo group) as described previously (Chen et al., 2009). In a cell viability assay, we found that transfection of MAFK alone could significantly increase cell viability from day 4 . However, co-transfection of MAFK and Wnt1 plasmids caused a significant increase in cell numbers from day 2 , with cell numbers approximately four-fold increased on day 4. IWR-1-endo decreased the highly enhanced cell proliferation induced by co-transfection of MAFK and Wnt1 plasmids (Figure 4A). We then performed a FACS assay to evaluate whether cell proliferation was enhanced due to re-distribution of the cell cycle. As shown in Figure 4B, the cell population in the G0/G1 stage was decreased after transfection of the MAFK plasmid; it was further decreased after co-transfection of both MAFK and Wnt1 plasmids. However, the cell population at the G0/G1 stage was increased compared with the MAFK+Wnt 1 group after IWR-1-endo was added with MAFK in MG63 cells. The altered cell percentage of the G0/G1 stage caused corresponding G2/M cell population changes in distinct groups. These data suggested that Wnt1-induced MAFK expression is critical for cell proliferation in MG63 cells, and that cell proliferation was enhanced potentially as a consequence of re-distribution of the cell cycle.

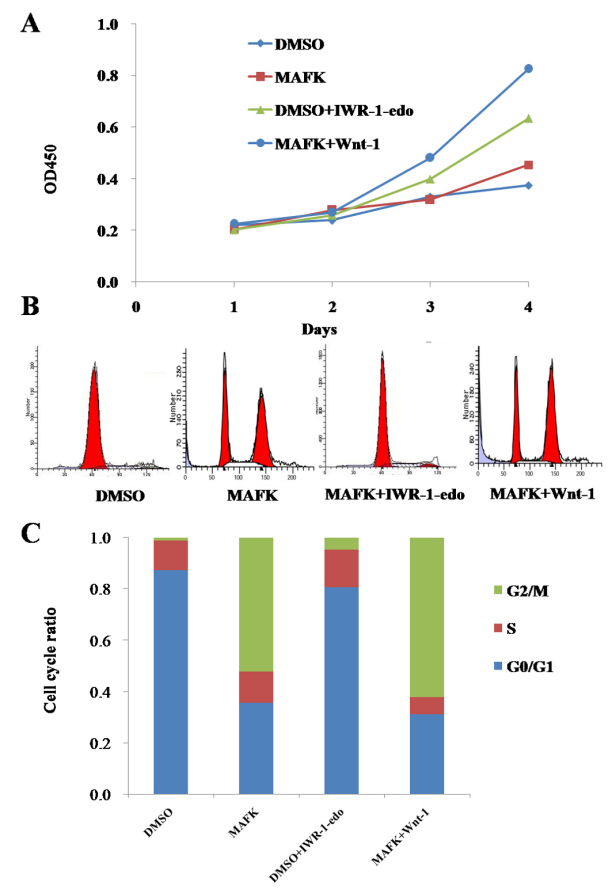

Figure 4. Wnt1-induced MAFK expression promoted cell proliferation in MG63 cells. A. Transfection of Wnt1 promoted MAFK-mediated cell proliferation in MG63 cells. Co-incubation with the Wnt signaling inhibitor, IWR-1endo, abolished Wnt1-induced effects on MAFK-mediated cell proliferation. $* \mathrm{P}<0.05$. B. C. Fluorescence-activated cell sorting assay showed that MAFK-mediated cell proliferation could be attributed to re-distribution of the cell cycle. 


\section{DISCUSSION}

Osteosarcoma is the most common malignant bone tumor in children and adolescents (Bielack et al., 2008; Siclari and Qin, 2010). Many patients experience tumor recurrence following treatment with current osteosarcoma therapy, which consists of chemotherapy and surgery (Zhong et al., 2014). However, targeted molecular therapy holds promise as a new avenue in human cancer treatments.

In this study, an enhancement effect of MAFK on human osteosarcoma MG63 cell proliferation was investigated. Initially, we observed that MAFK was expressed at low levels in osteosarcoma samples (Figure 1). However, when MG63 cells were transfected with an MAFK plasmid, cells exhibited significantly increased proliferation ability (Figure 4A, MAFK panel). The enhanced proliferation was, at least in part, attributed to re-distribution of the cell cycle (Figure 4B, MAFK panel). In addition, we investigated the possible mechanisms that contribute to MAFK-mediated cell proliferation in MG63 cells. Microarray analysis revealed that the Wnt signaling pathway was significantly linked to MAFK-mediated osteosarcoma development (Figure 2). More importantly, Wnt1 could induce expression of MAFK at both the mRNA and protein level (Figure 3). Induced expression of MAFK by Wnt1 could increase MAFK-promoted cell proliferation in MG63 cells, whereas the Wnt signaling inhibitor, IWR-1-endo, abolished the effects of Wnt1 on MAFK (Figure 3). Taken together, it could be concluded that MAFK might exert an enhancing effect on MG63 cell proliferation, which could be further induced by Wnt1. MAFK-mediated osteosarcoma development might be closely linked to the Wnt signaling pathway.

The Wnt signaling pathway consists of a family of highly conserved proteins that act as signal transducers for downstream genes that are involved in cell proliferation and growth (Hoang, 2012). Several Wnt ligands, receptors, and coreceptors are highly expressed in osteosarcoma cell lines, while Wnt inhibitors are downregulated (McQueen et al., 2011). In fact, the role of Wnt in the development of osteosarcoma has been widely reported (Brun et al., 2013; Yang and Zhang, 2013), and has aroused scientific interests worldwide. Wnt inhibitory factor 1 , an endogenous secreted Wnt pathway antagonist, was found to be epigenetically silenced in human osteosarcoma, and targeted disruption was shown to accelerate osteosarcomagenesis in mice (Kansara et al., 2009). These reports indicated that Wnt signaling might be potentially involved in osteosarcomagenesis. In our study, we provided evidence that the Wnt signaling pathway was closely linked to MAFK-mediated osteosarcoma development. On the one hand, the findings relating to the Wnt pathway have enriched our knowledge on how MAFK was involved in osteosarcoma proliferation. On the other hand, Wnt1-induced MAFK expression, which resulted in corresponding MG63 cell proliferation, was strongly supportive of the notion that Wnt could exhibit pro-tumor effect in osteosarcoma developments. The Wnt signaling inhibitor, IWR-1-endo, also efficiently inhibited the effects exerted on MG63 cells by Wnt1. Thus, we speculated that use of the Wnt pathway inhibitor might be a novel approach for development of osteosarcoma treatments.

MAFK was previously shown to interact with other transcription factors, especially Nrf2 (Ney et al., 1993; Itoh et al., 1997; Nguyen et al., 2000). However, interaction with Nrf2 was only observed in MAFK-mediated redox homeostasis (Dhakshinamoorthy and Jaiswal, 2000). In the development of osteosarcoma, there might be novel mechanisms by which MAFK initiates osteosarcomagenesis. Our study was limited to the role of Wnt1 as an upstream gene that could induce MAFK expression. The downstream regulatory network that 
contributes to MAFK-mediated osteosarcomagenesis remains to be elucidated. In light of the data shown in Figure 4B, factors that arrest the cell cycle, particularly in the G0/G1 stage, might be possibly targeted by MAFK in MAFK-mediated osteosarcomagenesis. However, more work needs to be done in this area in the future.

In summary, we studied the effect of MAFK on human osteosarcoma MG63 cells and the role of Wnt1 on MAFK-mediated MG63 cell proliferation. We also presented evidence that the Wnt signaling pathway might be closely linked to MAFK-mediated osteosarcoma development by using microarray analysis. Our findings provide evidence that targeted therapy against MAFK or Wnt signaling inhibitors might be promising for the development of human osteosarcoma treatments.

\section{ACKNOWLEDGMENTS}

The authors thank Zhi-Li and Bin-Qiao of Pubsci Company for their technical support.

\section{REFERENCES}

Bielack SS, Carrle D, Hardes J, Schuck A, et al. (2008). Bone tumors in adolescents and young adults. Curr. Treat. Options Oncol. 9: 67-80.

Blank V, Knoll JH and Andrews NC (1997). Molecular characterization and localization of the human MAFG gene. Genomics 44: 147-149.

Brand M, Ranish JA, Kummer NT, Hamilton J, et al. (2004). Dynamic changes in transcription factor complexes during erythroid differentiation revealed by quantitative proteomics. Nat. Struct. Mol. Biol. 11: 73-80.

Broadhead ML, Clark JC, Myers DE, Dass CR, et al. (2011). The molecular pathogenesis of osteosarcoma: a review. Sarcoma 2011: 959248.

Brun J, Dieudonne FX, Marty C, Muller J, et al. (2013). FHL2 silencing reduces Wnt signaling and osteosarcoma tumorigenesis in vitro and in vivo. PLoS One 8: e55034.

Chen B, Dodge ME, Tang W, Lu J, et al. (2009). Small molecule-mediated disruption of Wnt-dependent signaling in tissue regeneration and cancer. Nat. Chem. Biol. 5: 100-107.

Chou AJ and Gorlick R (2006). Chemotherapy resistance in osteosarcoma: current challenges and future directions. Expert Rev. Anticancer Ther. 6: 1075-1085.

Chou AJ, Geller DS and Gorlick R (2008). Therapy for osteosarcoma: where do we go from here? Paediatr. Drugs 10: 315-327.

Dhakshinamoorthy S and Jaiswal AK (2000). Small maf (MafG and MafK) proteins negatively regulate antioxidant response element-mediated expression and antioxidant induction of the $\mathrm{NAD}(\mathrm{P}) \mathrm{H}:$ Quinone oxidoreductase1 gene. J. Biol. Chem. 275: 40134-40141.

Francastel C, Magis W and Groudine M (2001). Nuclear relocation of a transactivator subunit precedes target gene activation. Proc. Natl. Acad. Sci. U. S. A. 98: 12120-12125.

Ghosh M, Brancato SJ, Agarwal PK and Apolo AB (2014). Targeted therapies in urothelial carcinoma. Curr. Opin. Oncol. 26: 305-320.

Gorjanacz M (2014). Nuclear assembly as a target for anti-cancer therapies. Nucleus 5: 47-55.

Hoang BH (2012). Wnt, osteosarcoma, and future therapy. J. Am. Acad. Orthop. Surg. 20: 58-59.

Itoh K, Chiba T, Takahashi S, Ishii T, et al (1997). An Nrf2/small Maf heterodimer mediates the induction of phase II detoxifying enzyme genes through antioxidant response elements. Biochem. Biophys. Res. Commun. 236: 313-322.

Kannan MB, Solovieva V and Blank V (2012). The small MAF transcription factors MAFF, MAFG and MAFK: current knowledge and perspectives. Biochim. Biophys. Acta 1823: 1841-1846.

Kansara M, Tsang M, Kodjabachian L, Sims NA, et al. (2009). Wnt inhibitory factor 1 is epigenetically silenced in human osteosarcoma, and targeted disruption accelerates osteosarcomagenesis in mice. J. Clin. Invest. 119: 837-851.

McQueen P, Ghaffar S, Guo Y, Rubin EM, et al. (2011). The Wnt signaling pathway: implications for therapy in osteosarcoma. Expert Rev. Anticancer Ther. 11: 1223-1232.

Ney PA, Andrews NC, Jane SM, Safer B, et al. (1993). Purification of the human NF-E2 complex: cDNA cloning of the hematopoietic cell-specific subunit and evidence for an associated partner. Mol. Cell Biol. 13: 5604-5612. 
Nguyen T, Huang HC and Pickett CB (2000). Transcriptional regulation of the antioxidant response element. Activation by Nrf2 and repression by MafK. J. Biol. Chem. 275: 15466-15473.

Nishikawa K, Nakashima T, Takeda S, Isogai M, et al. (2010). Maf promotes osteoblast differentiation in mice by mediating the age-related switch in mesenchymal cell differentiation. J. Clin. Invest. 120: 3455-3465.

Nishizawa M, Kataoka K, Goto N, Fujiwara KT, et al. (1989). v-maf, a viral oncogene that encodes a "leucine zipper" motif. Proc. Natl. Acad. Sci. U. S. A. 86: 7711-7715.

Okita Y, Kamoshida A, Suzuki H, Itoh K, et al. (2013). Transforming growth factor-beta induces transcription factors MafK and Bach1 to suppress expression of the heme oxygenase-1 gene. J. Biol. Chem. 288: 20658-20667.

Siclari VA and Qin L (2010). Targeting the osteosarcoma cancer stem cell. J. Orthop. Surg. Res. 5: 78.

Siegel HJ and Pressey JG (2008). Current concepts on the surgical and medical management of osteosarcoma. Expert Rev. Anticancer Ther. 8: 1257-1269.

Trent JC (2008). Rapid evolution of the biology and treatment of sarcoma. Curr. Opin. Oncol. 20: 393-394.

Vinson C, Acharya A and Taparowsky EJ (2006). Deciphering B-ZIP transcription factor interactions in vitro and in vivo. Biochim. Biophys. Acta 1759: 4-12.

Yang J and Zhang W (2013). New molecular insights into osteosarcoma targeted therapy. Curr. Opin. Oncol. 25: 398-406.

Zhong L, Wang Y, Li W, Gu J, et al. (2014). Heme oxygenase-1 silencing increases the sensitivity of human osteosarcoma MG63 cells to arsenic trioxide. Mol. Cell Biochem. 392: 135-144. 